\title{
mIOROSCOPY IOI
}

We appreciate the response to this publication feature and welcome all contributions. Contributions may be sent to our Technical Editors: José A. Mascorro, jmascor@hotmail.com or Phil Oshel, oshellpe@cmich.edu

\section{Freezing Biological Samples}

\section{Charles W. Scouten \& Miles Cunningham myNeuroLab.com \\ St.Louis,MO cwscouten@myneurolab.com}

Freezing damages cell membranes and reduces the histological readability of biological specimens. This is a discussion of why, the consequences, and how to minimize or avoid the damage introduced when using freezing as the means to harden biological tissue in order to cut thin sections for histology.

Pure water can exist in the solid state in three forms. Two forms are crystalline: one with a hexagonal lattice and the other with a cubic lattice. The third form capitalizes on the fact that water can be frozen so rapidly that it does not have time to form a crystal lattice and remains amorphous (vitreous form). Crystal formation is responsible for the expansion of water as it freezes (a property unique of water), which creates specimen preparation artifacts. Vitreous ice does not expand upon solidification. This makes it the only desirable form of ice appropriate for biological specimens.

Slow freezing promotes ice crystal formation, and thus expansion. Expansion of the water content in specimens caused by freezing stretches and penetrates cell membranes. Biological materials are poor thermal conductors. It is therefore likely that thermal gradients will exist as the sample is frozen, and some ice crystals will form in the interior of any piece of tissue over $10 \mathrm{~mm}$ from the cold source. Placed on a cold surface to freeze, specimens will freeze slowly and will thus likely form crystals, especially in parts most distant from the freezing surface. When sectioned, slowly frozen tissue with large crystal formation will have the well-known "swiss cheese" morphology consisting of multiple holes and lost cell contents, to varying degrees, depending on the degree and size of crystal formation.

Note that it is the rate of freezing, not the end-temperature that is critical here. Rapid freezing is a function of the amount of specimen surface in contact with the cold source, the volume of water within the specimen, the starting temperature of the water (specimen), and how cold the source is. Ideally, the cold source is at an extremely cold temperature and arranged to contact all the specimen's surfaces.

However, even if a specimen is successfully quick frozen in the vitreous form, it may not stay that way. Vitreous ice is an unstable state. Above $-121^{\circ} \mathrm{C}$, amorphous ice begins to gradually restructure as cubic ice, and expand. Above $-80^{\circ} \mathrm{C}$, cubic ice begins to restructure as hexagonal ice, and expands again. Fortunately, this is a slow conversion process, depending on temperature gradients, and the purity of the water.

In most common applications, tissue must be warmed above the snap freezing temperature in order to section it $\left(-9\right.$ to $-19^{\circ} \mathrm{C}$ for brain, but depending on the tissue type). Otherwise it is too cold to cut, and shatters when bent by the knife bevel. The visible symptom of cutting too cold is horizontal "stutters" or shatter marks. The sectioning should begin as soon as the tissue is warm enough to cut. Tissue should never be left overnight or for long periods in a cryostat, even is there is no defrost cycle. The conversion from vitreous ice to large hexagonal crystals will begin. Crystals will expand the ice, and damage cells.

The quickest way to freeze a specimen is by immersion in a liquid-creating the most surface contact. The second best method is to place the specimen on a pre-chilled metal pedestal and quickly surround the tissue with powdered dry ice-powdered to maximize surface contact.

Liquid nitrogen is one of the coldest liquids routinely available. It does not mix with tissue. These properties would seem to make it an ideal freezing medium. However, liquid nitrogen has an extremely low specific heat constant. The result is that it boils locally and copiously at the contact point with even a small piece of warmer tissue. One consequence is that a vapor barrier, consisting of a layer of nitrogen gas, can build up next to the tissue, and insulate and greatly slow penetration of cold into the tissue, and thus slow the rate of freezing in an unpredictable fashion. As a result, it often happens that the outer layer of tissue is quick frozen and vitreous, but when the vapor barrier is formed, the cold temperature penetrates into the specimen more slowly, and the inner part of the block freezes slower. Crystal formation expands the tissue inside the frozen shell, and the entire block cracks. A cracked block indicates that at least the innermost tissue has been frozen crystalline.

Various other liquids are used to give more consistent and reliable results, most notably isopentane cooled to $-80^{\circ} \mathrm{C}$. Isopentane does not form a vapor barrier on the specimen surface, and if the tissue is little more than a cubic centimeter or less in volume, the specimen's water content will freeze all in the vitreous form. Full immersion in the liquid is necessary for maximum surface contact, and harmless to the tissue. Movement through the liquid is helpful.

Another solution is to permeate or perfuse the tissue with glycerine, polyethylene glycol (antifreeze), glycerol, or sucrose solutions. These will disrupt crystal formation, and lower the freezing temperature. Sucrose at $30 \%$ in phosphate buffer is commonly used, but only with well fixed tissue, after fixation, as the osmolarity would shrivel unfixed tissue.

\section{Reference}

Jongebloed, W.L., Stokroos, D., Kalicharan, D., and Van der Want, J.J.L. Is Cryopreservation Superior Over Tannic Acid/Arginine/Osmium Tetroxide non-Coating Preparation in Field Emission Scanning Electron Microscopy. Scanning Microscopy 13: 93-109, 1999

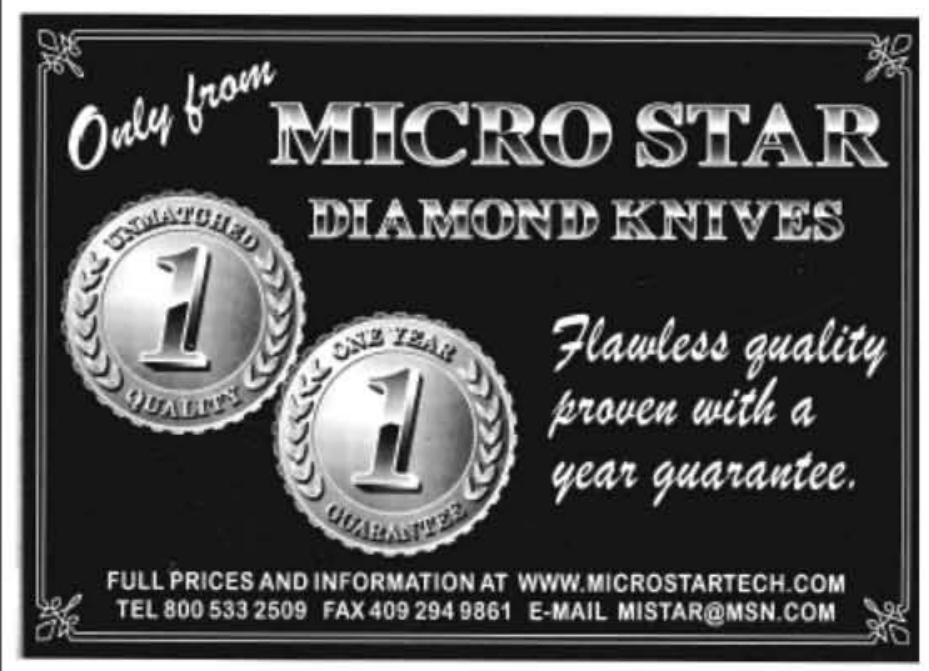

